\title{
A multiparametric approach to improve the prediction of response to immunotherapy in patients with metastatic NSCLC
}

\author{
Marzia Del Re ${ }^{1}$ - Federico Cucchiara ${ }^{1}$ - Eleonora Rofi ${ }^{1}$. Lorenzo Fontanelli ${ }^{1}$ - lacopo Petrini ${ }^{2} \cdot$ Nicole Gri $^{3}$. \\ Giulia Pasquini ${ }^{2} \cdot$ Mimma Rizzo $^{3} \cdot$ Michela Gabelloni $^{4} \cdot$ Lorenzo Belluomini $^{5} \cdot$ Stefania Crucitta ${ }^{1} \cdot$ Raffaele Ciampi $^{6}$. \\ Antonio Frassoldati ${ }^{5} \cdot$ Emanuele Neri $^{4} \cdot$ Camillo Porta $^{3,7,8} \cdot$ Romano Danesi $^{1}(\mathbb{C}$
}

Received: 2 July 2020 / Accepted: 23 November 2020 / Published online: 14 December 2020

(c) The Author(s) 2020

\begin{abstract}
Background It is still unclear how to combine biomarkers to identify patients who will truly benefit from anti-PD-1 agents in NSCLC. This study investigates exosomal mRNA expression of PD-L1 and IFN- $\gamma$, PD-L1 polymorphisms, tumor mutational load (TML) in circulating cell-free DNA (cfDNA) and radiomic features as possible predictive markers of response to nivolumab and pembrolizumab in metastatic NSCLC patients.

Methods Patients were enrolled and blood $(12 \mathrm{ml})$ was collected at baseline before receiving anti-PD-1 therapy. Exosomederived mRNA and cfDNA were extracted to analyse PD-L1 and IFN- $\gamma$ expression and tumor mutational load (TML) by digital droplet PCR (ddPCR) and next-generation sequencing (NGS), respectively. The PD-L1 single nucleotide polymorphisms (SNPs) c.-14-368 T > C and c. $* 395 \mathrm{G}>\mathrm{C}$, were analysed on genomic DNA by Real-Time PCR. A radiomic analysis was performed on the QUIBIM Precision ${ }^{\circledR} \mathrm{V} 3.0$ platform.

Results Thirty-eight patients were enrolled. High baseline IFN- $\gamma$ was independently associated with shorter median PFS (5.6 months vs. not reached $p=0.0057$ ), and levels of PD-L1 showed an increase at 3 months vs. baseline in patients who progressed $(p=0.01)$. PD-L1 baseline levels showed significant direct and inverse relationships with radiomic features. Radiomic features also inversely correlated with PD-L1 expression in tumor tissue. In subjects receiving nivolumab, median PFS was shorter in carriers of c.*395GG vs. c. $* 395 \mathrm{GC} / \mathrm{CC}$ genotype (2.3 months vs. not reached, $p=0.041)$. Lastly, responders had higher non-synonymous mutations and more links between co-occurring genetic somatic mutations and ARID1A alterations as well.

Conclusions A combined multiparametric approach may provide a better understanding of the molecular determinants of response to immunotherapy.

Keywords NSCLC $\cdot$ Liquid biopsy $\cdot$ Biomarkers $\cdot$ Radiomics $\cdot$ Immunotherapy

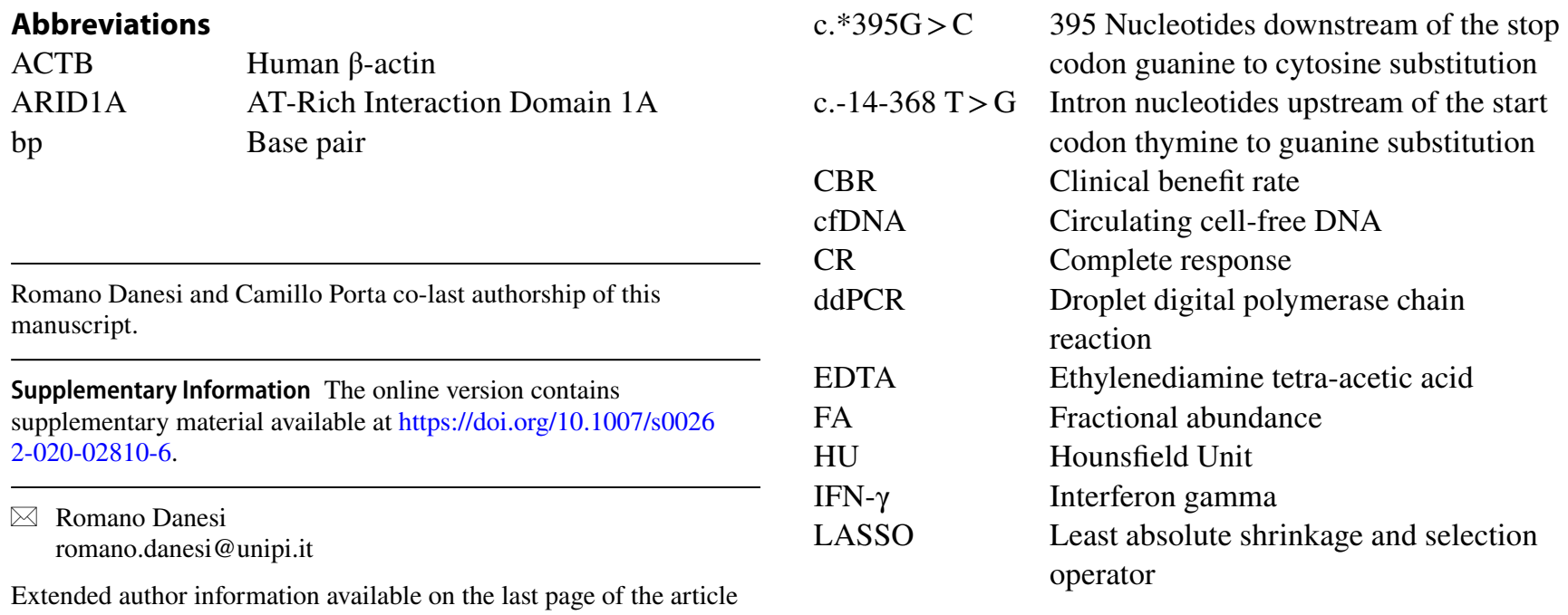




$\begin{array}{ll}\text { MMR } & \text { Mismatch repair } \\ \text { MSI } & \text { Microsatellite instability } \\ \text { NGS } & \text { Next-generation sequencing } \\ \text { NSCLC } & \text { Non-small cell lung cancer } \\ \text { ORR } & \text { Overall response rate } \\ \text { PD } & \text { Progression disease } \\ \text { PD-1 } & \text { Programmed cell death protein-1 } \\ \text { PD-L1 } & \text { Programmed cell death protein-ligand 1 } \\ \text { PFS } & \text { Progression free survival } \\ \text { PolyPhen } & \text { Polymorphism phenotyping } \\ \text { PR } & \text { Partial responses } \\ \text { RECIST } & \text { Response evaluation criteria in solid } \\ & \text { tumors } \\ \text { ROC } & \text { Receiver operating characteristic } \\ \text { SD } & \text { Stable disease } \\ \text { SIFT } & \text { Sorting intolerant from tolerant } \\ \text { SNPs } & \text { Single nucleotide polymorphisms } \\ \text { SNV } & \text { Single-nucleotide variant } \\ \text { TML } & \text { Tumor mutational load } \\ \text { TPS } & \text { Tumor proportional score } \\ \text { VOI } & \text { Volume of interest }\end{array}$

\section{Background}

Anti-PD-1 therapies significantly improved the prognosis in a subset of patients affected by NSCLC. Despite several predictive biomarkers have been proposed, it is still unclear how to manage them in an integrated model to improve prediction power. PD-L1 assessment in tumor tissue has been widely used to identify patients who will benefit from immune-checkpoint inhibition; however, intratumor heterogeneity may cause false negative results [1] and patients with low PD-L1 expression may also take advantage from pembrolizumab [2]. Moreover, tumor phenotype, including PD-L1 expression, may vary throughout time in response to alterations in the tumor microenvironment and following the clonal selection induced by treatments [3-6]. For these reasons, other markers, such us PD-1/PD-L1 polymorphisms, tumor immune infiltrate, mutational load and microsatellite instability have been considered [7-9]. Moreover, several other mediators of the immune response have been investigated, such as IFN- $\gamma$, which has a pleiotropic activity on the immune system and can alter the expression of PD-L1 [10], albeit its role in tumor progression and immunotherapy response is still controversial [11]. To overcome spatial and temporal tumor heterogeneity, cell free tumor DNA and exosomes, due to their involvement in immune signalling, reprogramming of surrounding cells and immune escape, are gaining attention [12-15]. In addition to this, the concept that biomedical images contain information reflecting tumor molecular aberrations is nowadays raising, and radiomic analysis is being used to identify predictive biomarkers of response to treatments $[16,17]$. For these reasons, the present study was aimed at integrating the evaluation of: (1) PD-L1 and IFN- $\gamma$ mRNA expression in plasma-derived exosomes; (2) selected PD-L1 gene variants (i.e. c.-14$368 \mathrm{~T}>\mathrm{G}$ and c.*395G $>\mathrm{C}$ ); (3) tumor mutational load (TML) on cfDNA, and (4) radiomic analysis to identify predictive biomarkers of response to anti-PD-1 therapy.

\section{Methods}

\section{Patients}

Patients affected by locally advanced or metastatic NSCLC given nivolumab or pembrolizumab as per approved schedule were enrolled in the present study. Blood samples were drawn from each patient for the analysis of (1) exosomal mRNA levels of PD-L1 and IFN- $\gamma$ at time 0 (baseline) and after 3 months of treatment, (2) selected germinal PD-L1 polymorphisms and (3) tumor mutational load (TML) in circulating free DNA (cfDNA). PD-L1 immuno-histochemistry assessment in tumor tissue was collected in selected cases, accordingly with laboratories procedures. Complete (CR) and partial response (PR), disease stabilisation (SD) and disease progression (PD) were defined following RECIST (v. 1.1) criteria. CT scans were collected at baseline for radiomic analysis (see below). A written consent form was obtained from all patients; the study was approved by the local Ethics Committee and performed in accordance with the provisions established by the Helsinki Declaration.

\section{Exosomes isolation and measurement of PD-L1 and IFN- $\gamma$ mRNA}

A blood sample of $12 \mathrm{ml}$ was collected in EDTA tubes and centrifuged for $10 \mathrm{~min}$ at $1900 \mathrm{~g}$ within $2 \mathrm{~h}$. Exosomes were isolated from plasma and RNA was extracted, as previously described [18]. Expression of PD-L1 and IFN- $\gamma$ was assed via ddPCR (Bio-Rad, Hercules, CA) with respect to human $\beta$-actin (ACTB) as internal control, as previously described [4]. The values reported are expressed as fractional abundance (FA, \%), that is the proportion of the number of copies $/ \mathrm{ml}$ of the investigated protein-coding exosomal mRNAs in the total of the commonly detected exosomal mRNAs, including also $\beta$-actin (ACTB) as housekeeping gene, calculated by the QuantaSoft ${ }^{\mathrm{TM}}$ software (Bio-Rad, Hercules, CA).

\section{Analysis of PD-L1 germline polymorphisms}

Germline DNA was extracted from $200 \mu \mathrm{l}$ of peripheral blood (EZ1 Extractor; Qiagen, Valencia, CA) for the analysis of the PD-L1 single nucleotide polymorphisms (SNPs) c. $* 395 \mathrm{G}>\mathrm{C}$ and c. $-14-368 \mathrm{~T}>\mathrm{C}$, selected on the basis of a previous publication $[9,19]$, by a real-time PCR using the 
TaqMan ${ }^{\circledR}$ SNP Genotyping Assay (ThermoFisher, Carlsbad, CA).

\section{TML analysis on cfDNA}

A blood sample of $6 \mathrm{ml}$ was collected in EDTA tubes and centrifuged for $10 \mathrm{~min}$ at $1900 \mathrm{~g}$ within $2 \mathrm{~h}$. cfDNA was extracted from $3 \mathrm{ml}$ of plasma and DNA was eluted in $50 \mu \mathrm{l}$ of buffer, as previously published [20]. TML analysis was performed on the Ion S5 XL System NGS platform, using the Oncomine Tumor Mutation Load Assay (ThermoFisher, Carlsbad, CA). The filtered variants were examined using the Integrative 201 Genomic Viewer IGV tool to check their quality level and confirm the presence of the variant of interest. To explore the relationship between the quality of genetic profiles and TML, all genes and relative calling mutations were evaluated according to their involvement in NSCLC pathogenesis, immune system, cell cycle, and immunotherapy response. For each SNV (single-nucleotide variant) occurring in the exonic region, an estimation of its putative damaging effect on the resulting protein was scored using Grantham, SIFT and PolyPhen criteria [21]. Finally, a computational approach was attempted [22, 23]. Starting from Milgram's basic small-world concept [22] a custommade MATLAB ${ }^{\circledR}$ script (The Math Works Inc., Natick, MA) was endeavored to investigate possible relationships between genes included in the Oncomine panel. For each gene the total number of different mutations was considered, weighed by the number of mutations with Polyp hen $\geq 0.85$ (deleterious power) and a Watts-Strogatz graph [23] was plotted.

\section{Radiomic analysis}

Patients who undergone to CT imaging for lung cancer staging and met homogeneity criteria for image acquisition parameters [24], were retrospectively enrolled in a radiomics analysis evaluating 25 radiomics features from the entire primary tumor lesion at baseline (Supplementary Table S1). Scan protocol homogeneity criteria included $120 \mathrm{kV}$ tube voltage, a field of view between 36 and $40 \mathrm{~cm}, 1.5-2 \mathrm{~mm}$ slice thickness and a standard/soft tissue convolution kernel. Only non-contrast CT images were used for radiomics analysis. CT scan were acquired at baseline of immunotherapy, either first-line pembrolizumab or nivolumab as subsequentline. The primary tumor site was manually contoured slice by slice on axial CT images using a lung window setting (width, $1500 \mathrm{HU}$; level, - $600 \mathrm{HU}$ ) by a radiologist experienced in lung cancer imaging, and then independently validated by another radiologist assessor. Radiomic analysis was then performed on the volume of interest (VOI) via the QUIBIM Precision ${ }^{\circledR}$ V3.0 platform (QUIBIM SL, Valencia, Spain) [25]. Lastly, a statistical method was used to avoid a redundancy of information [26] and to select only the most distinctive radiomic features (Supplementary Table S2).

\section{Statistical analysis}

Categorical variables were described by absolute and relative frequencies while quantitative factors by median and range. To compare quantitative with categorical variables the Mann-Whitney test was performed, while the Wilcoxon's test was used to assess paired data. The median cut-off value for analysis of PD-L1 and IFN- $\gamma$ was calculated by the Receiver Operating Characteristic (ROC) curve analysis to differentiate patients with response and no response to ICIs. Since an overall survival (OS) advantage was difficult to detect due to the small sample size and the short follow-up, progression free survival (PFS), overall response rate (ORR) and clinical benefit rate (CBR) were investigated [27, 28]. Moreover, CBR and PFS are generally based on objective and quantitative assessments, including the measurement of stable disease, and are not affected by crossover or subsequent therapies. PFS was defined as the time from treatment start to PD or death. ORR was defined as the proportion of patients achieving CR and PR. CBR was defined as the proportion of patients achieving $\mathrm{CR}, \mathrm{PR}$ or $\mathrm{SD}$ for at least 24 weeks. Log-rank test was used to evaluate differences between curves and hazard ratio was calculated using Cox model to compare cumulative risks. Pearson's correlation coefficient was used to assess the correlation among all radiomic features, and between radiomic features and available molecular data. Lastly, a radiomic signature was calculated based on the sum of the features correlating with tumor molecular data, weighted by their corresponding maximumlikelihood fitted coefficients for the least absolute shrinkage and selection operator (LASSO) regression model. A 11-fold cross validation was performed for this purpose. Logistic regression model test (Cox-Snell's $R^{2}$ ) and receiver operating characteristic (ROC) curve analysis was computed to estimate diagnostic performance of such signatures, alone and combined with the other biomarkers analysed. Differences were considered significant at $p<0.05$. All statistical analyses were performed with SPSS version 24 (SPSS Inc. SPSS $^{\circledR}$ Chicago, IL, USA) or MatLab ${ }^{\circledR}$ software (version R2019a; MatLab, The Math Works Inc., Natick, MA).

\section{Results}

\section{Patients' characteristics}

A total of 38 NSCLC patients were enrolled in the study (Table 1). Twenty-five patients received nivolumab as second (68\%) or higher line of treatment (32\%), while 13 patients received pembrolizumab as first line treatment. 
At the first radiological assessment 13 patients (34.2\%) obtained a PR, 8 (31.6\%) SD and 17 (44.8\%) PD. ORR was $34 \%$, slightly different amongst the two cohorts (53\% vs. $24 \%$ for pembrolizumab and nivolumab, respectively), CBR was $66 \%$ ( $85 \%$ vs. $56 \%$ for pembrolizumab and nivolumab, respectively).

\section{Association between mRNA expression of PD-L1 and IFN- $\gamma$ and clinical outcome}

Median FA at baseline was $0.3 \%$ for PD-L1 and $4.1 \%$ for IFN- $\gamma$ (overall population, 38 patients). PFS was 11 vs. 16.2 months in patients with PD-L1 FA of $<0.3 \%$ vs. $\geq 0.3 \%$, respectively ( $p=0.75$; Fig. 1a). PFS was 5.6 months vs. not reached in patients with baseline FA of IFN- $\gamma$ of $\geq 4.1 \%$ vs. $<4.1 \%$, respectively ( $p=0.0057$; Fig. 1b). Comparing PD-L1 at baseline vs. 3 months of treatment, the FA was significantly increased in patients with PD vs. PR $+\mathrm{SD}(p=0.01$; Fig. 1c). Of note, considering IFN- $\gamma$ baseline levels, the FA was significantly higher in patients who progressed within 3 months compared to patients with $\mathrm{PR}+\mathrm{SD}(p=0.04$; Fig. $1 \mathrm{~d})$.

\section{Association between PD-L1 polymorphism and clinical outcome}

c. $-14-368 \mathrm{~T}>\mathrm{G}$ and c.*395G $>$ C PD-L1 polymorphisms were obtained in 32 out of 38 patients ( 20 patients treated with nivolumab and 12 with pembrolizumab). The absolute and relative frequencies are reported in Table 2. In the cohort treated with nivolumab, median PFS was significantly shorter in patients with c.*395GG vs. those carrying the c.*395GC/CC genotype (2.3 months vs. not reached, $p=0.041$; Fig. 2). No significant association between c.*395G $>$ C genotypes and PFS was observed in cohort of patients given pembrolizumab and in the overall population. The c.-14-368 T > G genotype was not correlated with PFS neither in the overall population nor in the cohorts treated with nivolumab or pembrolizumab alone.

\section{TML and mutated gene network}

TML analysis on cfDNA was available only in cfDNA samples showing a sufficient concentration of 150-300 bp DNA. PD was associated with lower mutation load compared to patients who achieved a PR (Supplementary Table S3). Furthermore, for all the exonic SNVs of each patient, the relative median values of Grantham, PolyPhen and SIFT scores were obtained. Subjects who underwent PR had higher
Table 1 Clinical characteristics of patients

\begin{tabular}{|c|c|c|c|}
\hline & $\begin{array}{l}\text { Overall popula- } \\
\text { tion }(n=38)\end{array}$ & Nivolumab $(n=25)$ & Pembrolizumab $(n=13)$ \\
\hline Age, mean (range) & $68(44-85)$ & $72(52-85)$ & $65.5(44-74)$ \\
\hline \multicolumn{4}{|l|}{ Sex, $n(\%)$} \\
\hline Male & $22(57.9)$ & $13(52)$ & $9(69.2)$ \\
\hline Female & $16(42.1)$ & $12(48)$ & $4(30.8)$ \\
\hline \multicolumn{4}{|l|}{ Smoking habits, $n(\%)$} \\
\hline Yes/former & $34(89.5)$ & $22(88)$ & $12(92.3)$ \\
\hline Never & $4(10.5)$ & $3(12)$ & $1(7.7)$ \\
\hline \multicolumn{4}{|l|}{ ECOG PS, $n(\%)$} \\
\hline $0-1$ & $38(100)$ & $25(100)$ & $13(100)$ \\
\hline$\geq 2$ & 0 & 0 & 0 \\
\hline \multicolumn{4}{|l|}{ Stage, $n(\%)$} \\
\hline III & 0 & 0 & 0 \\
\hline IV & $38(100)$ & $25(100)$ & $13(100)$ \\
\hline Sites of disease, mean (range) & $2(1-5)$ & $2.5(1-5)$ & $2(1-5)$ \\
\hline \multicolumn{4}{|l|}{ Site of disease } \\
\hline Bone, $n(\%)$ & $9(23.7)$ & $6(24)$ & $3(23.1)$ \\
\hline Visceral, $n(\%)$ & $6(15.8)$ & $5(20)$ & $1(7.7)$ \\
\hline Central nervous system, $n(\%)$ & $2(5.3)$ & $2(8)$ & 0 \\
\hline \multicolumn{4}{|l|}{ Line of therapy, $n(\%)$} \\
\hline I & $8(21.1)$ & 0 & $8(61.5)$ \\
\hline$\geq$ II & $30(78.9)$ & $25(100)$ & $5(38.5)$ \\
\hline
\end{tabular}

ECOG PS eastern cooperative oncology group performance status, $n$ number 
A

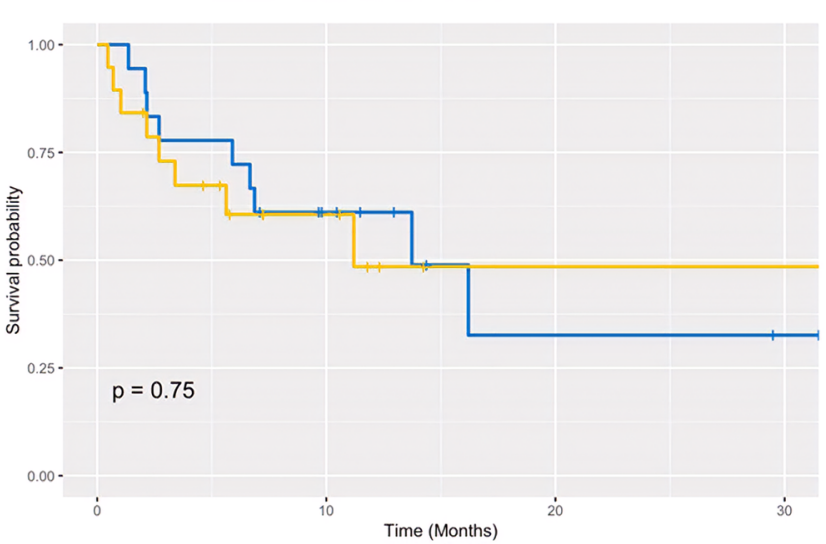

C
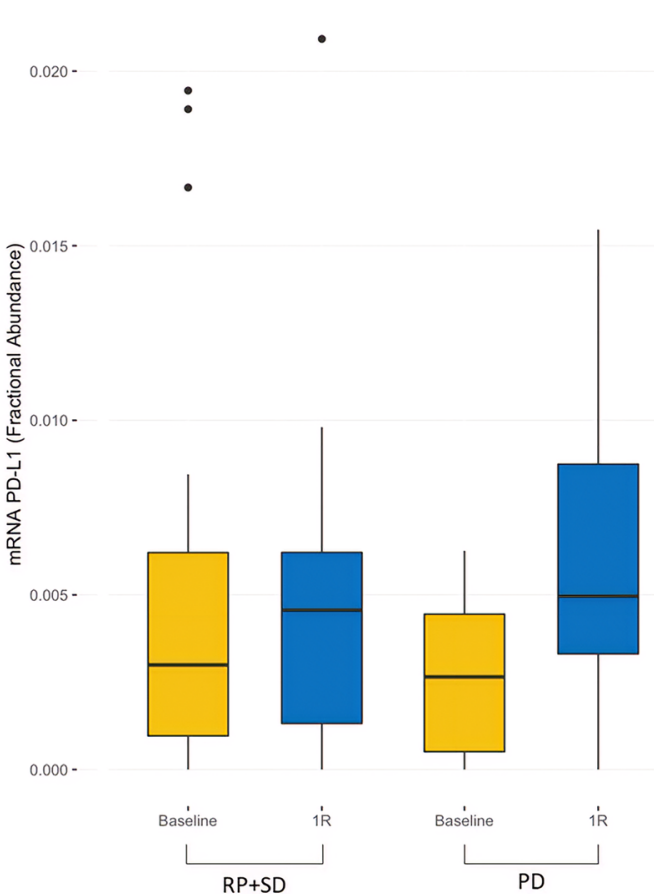

B

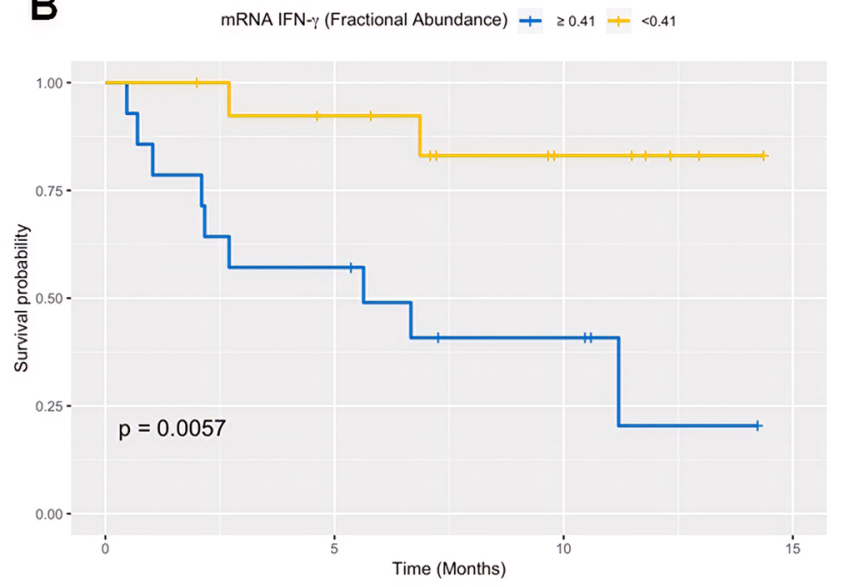

D

$$
0.35-
$$$$
0.30 \text { - }
$$

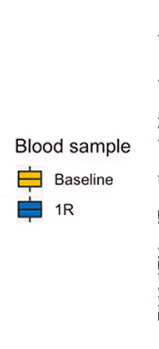

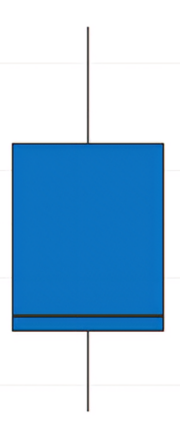

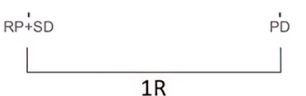

Clinical Response 官 $\mathrm{RP}+\mathrm{SD}$ 当 PD

Fig. 1 Kaplan-Meier PFS of 38 NSCLC patients stratified on the basis of fractional abundance of PD-L1 (a) and IFN- $\gamma$ (b). Comparison of fractional abundance of PD-L1 (c) and IFN- $\gamma(\mathbf{d})$ among patients with PR, SD and PD, at baseline and after first evaluation (1R)

Table 2 PD-L1 genotypes and clinical response at 3 months evaluation

\begin{tabular}{|c|c|c|c|c|c|c|}
\hline \multirow[t]{2}{*}{ Clinical response } & \multicolumn{3}{|c|}{ c. $* 395 \mathrm{G}>\mathrm{C}(n=32)$} & \multicolumn{3}{|c|}{ c. $-14-368 \mathrm{~T}>\mathrm{G}(n=32)$} \\
\hline & GG & GC & $\mathrm{CC}$ & TT & TG & GG \\
\hline $\mathrm{PR}, n(\%)$ & $6(18.8)$ & $6(18.8)$ & $1(3.2)$ & $5(15.6)$ & $6(18.8)$ & $2(6.2)$ \\
\hline $\mathrm{SD}, n(\%)$ & $3(9.4)$ & $4(12.5)$ & $1(3.2)$ & $5(15.6)$ & $3(9.4)$ & 0 \\
\hline $\mathrm{PD}, n(\%)$ & $8(25)$ & $3(9.4)$ & 0 & $4(12.5)$ & $5(15.6)$ & $2(6.2)$ \\
\hline
\end{tabular}

$n$ number, $T$ time, $P R$ partial response, $S D$ stable disease, $P D$ progression disease
Grantham, PolyPhen and SIFT mean values than the patient in $\mathrm{PD}(67.17 \pm 6.53$ vs. $50,0.01$ vs. $0,0.90 \pm 0.04$ vs. 0.80 , respectively).
Likewise, in the underlying architecture among mutated genes defining the TML, fewer connections were evident between mutated AT-Rich Interaction Domain 1A 


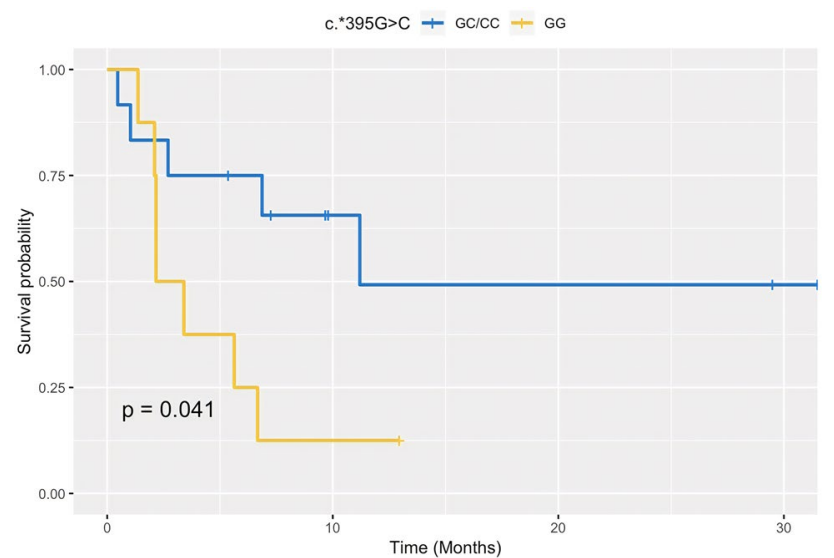

Fig. 2 Kaplan-Meier curve of PFS in 20 NSCLC patients treated with nivolumab according to the PD-L1 c. $* 395 \mathrm{G}>$ C polymorphism

(ARID1A), a gene involved in transcriptional regulation and DNA damage response, and the other genes in the patient who had PD with respect to those who achieved PR (Fig. 3).

\section{Radiomic results}

Radiomic analysis was performed in 11 subjects (Supplementary Fig. S1). FA of PD-L1 at baseline directly correlated with contrast $(p=0.96, p=0.003)$, dissimilarity $(p=0.92, p=0.008)$ and sum variance $(p=0.91, p=0.009)$, and inversely with entropy $(p=-0.95, p=0.004)$. Inverse correlations were also found between PD-L1 expression in tumor tissue with autocorrelation $(p=-0.83, p=0.04)$ and sum average ( $p=-0.82, p=0.05)$.

Notably, tissue expression of PD-L1 was variable; in $45 \%$ of subjects the PD-L1 tumor proportional score (TPS) was $<1 \%$, in $27.5 \%$ was in the $1-49 \%$ range and in $27.5 \%$ was $\geq 50 \%$. Two of them had levels $\geq 75 \%$.
No correlations were found between radiomic features and IFN- $\gamma$ FA. The radiomic signature comprising the abovementioned 6 features evidenced good capability with acceptable representativeness for predicting patients in PD vs. those who underwent PR or SD (Cox-Snell's $R^{2}=0.63$, $p<0.001)$. The optimal cut-point estimated from the ROC curve showed $85.71 \%$ sensitivity and $100 \%$ specificity, with the area under the curve of 0.96 . Combining the signature with the other biomarkers, representativeness improved (Cox-Snell's $R^{2}=0.72, p=0.02$ ) and ROC curve showed sensitivity and specificity of $100 \%$. A multiparametric analysis gave a better diagnostic performance than single parameters: with regard to clinical response, the sensitivity and specificity were, respectively, $75 \%$ vs. $57.14 \%$ for FA of PD-L1, $100 \%$ vs. $85.71 \%$ for FA of IFN- $\gamma$ and $71.43 \%$ vs. $75 \%$ for PD-L1 TPS cutoff of 50\% (Fig. 4).

\section{Discussion}

The availability of predictive biomarkers for immunotherapy response is still an important need in many solid tumors. Many efforts have been made to identify a reliable marker; however, except for the PD-L1 expression of patients candidate to first line treatment with pembrolizumab, no robust biomarkers have been identified. Even among NSCLC patients with PD-L1 expression $>50 \%$ treated with firstline pembrolizumab, clinical outcome resulted significantly improved in patients with a PD-L1 expression > 90\% [29]. While drivers such as EGFR, BRAF, ALK are HER2 dictate the choice of target-specific therapy, the same cannot be attributed to PD-L1 because it is dynamic, inducible, and disease-dependent. Therefore, it is reasonable that more than one biomarker is needed to select patients who will benefit or not from immunotherapy. Based on this hypothesis, in this study we considered exosomal mRNA expression of PD-L1 and IFN- $\gamma$, together with cfDNA-derived TML and radiomic

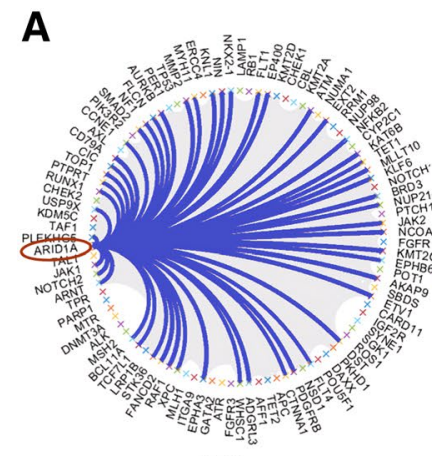

PD

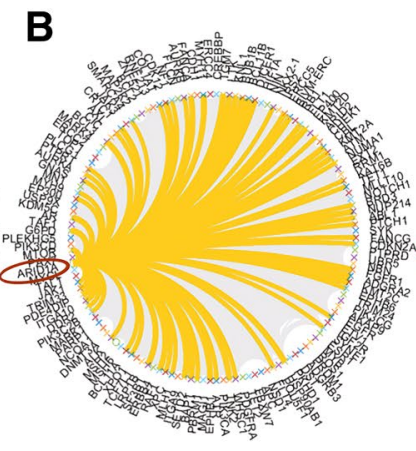

PR

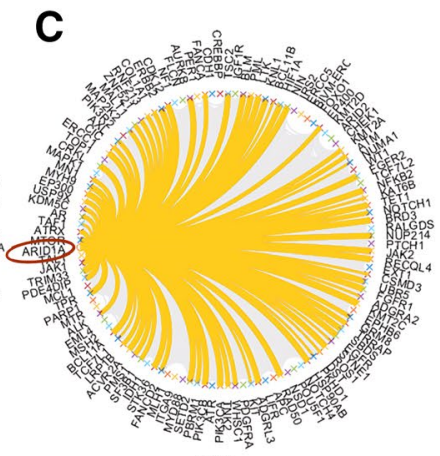

PR

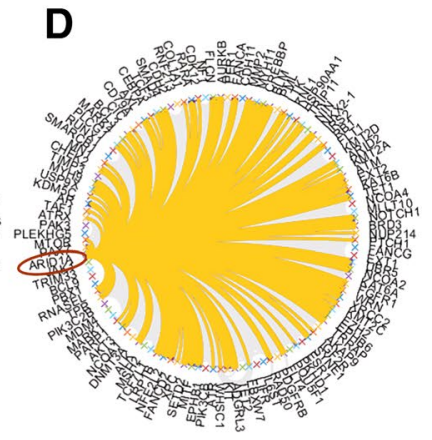

PR

Fig. 3 Watts-Strogatz graph showing the connections between ARID1A and other genes included in the TML NGS panel according to clinical response 
Fig. 4 Specificity and sensitivity of radiomic signature, PD-L1 and IFN- $\gamma$ FA and c. $* 395 \mathrm{G}>\mathrm{C}$ polymorphism

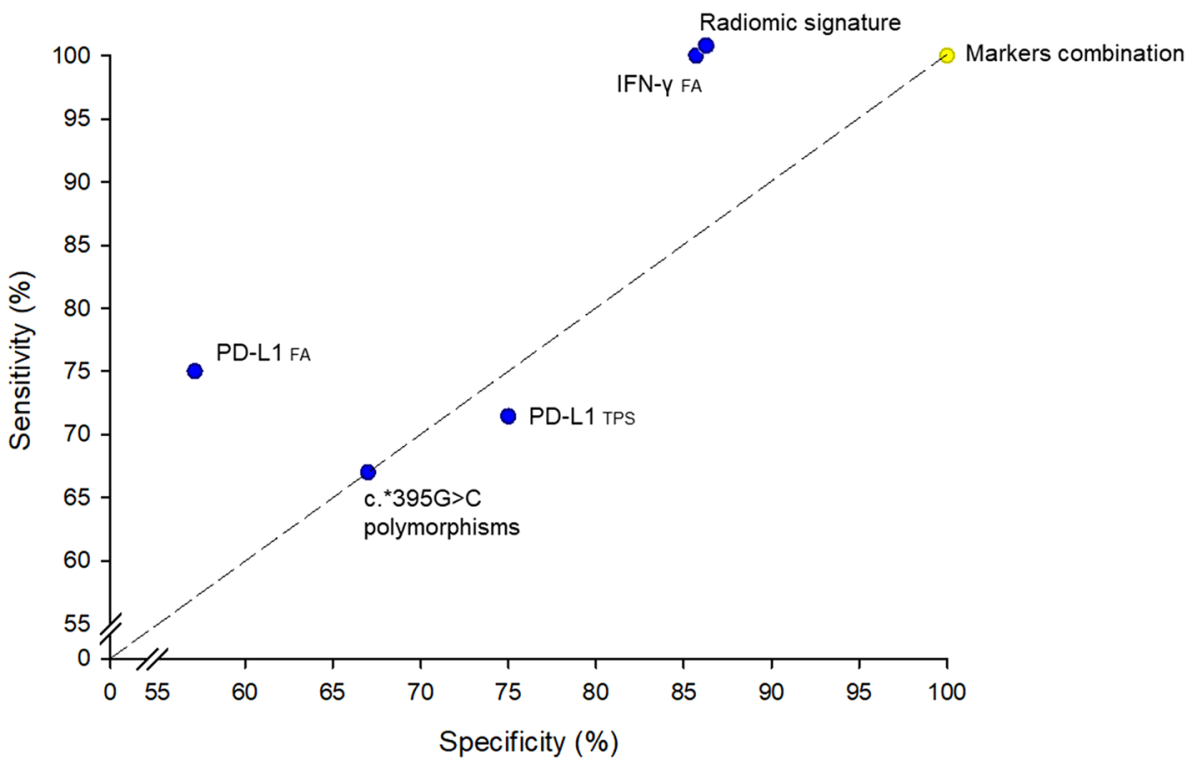

features as a possible pool of predictive markers of response to the anti-PD-1 agents nivolumab and pembrolizumab in NSCLC.

The baseline expression of IFN- $\gamma$ was significantly higher in patients who progressed. Indeed, although IFN- $\gamma$ has long been implicated as a central orchestrator of antitumor immune responses [30], mounting evidence [31-33] suggests that it may also have a pivotal role in immune evasion. IFN- $\gamma$ upregulates PD-L1 expression in cancer, stromal and myeloid cells, impairing immune response [34]. Both CD8 + T-cells and IFN- $\gamma$ are critical for antitumor immunity [35], but a prolonged IFN- $\gamma$ signaling in tumors, coordinates resistance to immune checkpoint inhibitors through a multigenic resistance program [36] independent of PD-L1 expression. Different studies evaluated the role of inflammatory cytokines as predictive biomarkers, although results are controversial [37]. The emerging picture of the immune landscape of NSCLC has provided evidence for an extremely high degree of complexity and heterogeneity [38]. Considering such heterogeneity, looking at only few markers (such as PD-L1) could be not robust enough. For these reasons, the development of a multiparametric approach is an emerging challenge to select patients more likely to respond. The feasibility to assess TML on cfDNA was evaluated, and a radiomic analysis has been conducted in this study. It is well known that tumors with high TML and responsive to immunotherapy may exhibit specific non-synonymous genetic alterations [39, 40]. For example, defects in MMR genes lead to MSI and could cause an increase in TML [39, 41]. In this study, albeit in a small cohort, patients who achieved a PR had higher amount of non-synonymous exonic mutations than the patient who underwent PD. Moreover, Grantham, SIFT and PolyPhen scores were higher in patients with $\mathrm{PR}$, indicating a possible biological condition consisting of numerous damaging mutations, compared with those found in the progressing patient. It is likely that an immunogenic phenotype may arise, leading to better responsiveness to immunotherapy. Of note, in the underlying architecture among mutated genes included in the NGS panel used to assess TML, patients who achieved a PR showed higher connections between mutated ARID1A gene and the others with respect to the one who underwent PD (Fig. 3). A pan-cancer analysis of ARID1A alterations [42] recently highlighted their important value as predictive biomarkers for immunotherapy. ARID1A alterations promote cancer iper-mutated phenotype [43, 44] and co-occurring specific genetic mutations in cancers with ARID1A alterations are detected [42]. Looking at differences in the number of ARID1A connections between PD and PR patients may not only suggests a possible novel marker to be considered in patients treated with immunotherapy, but also confirms the role of ARID1A in promoting immunogenicity. Currently, TML measurements are essentially performed using NGS in tissue biopsy and the cut-off can vary from 5 to $10 \%$ [45]. TML measurements from cfDNA is technically challenging, due to the low quantity and quality of cfDNA that can be extracted from plasma. Therefore, the identification of the cut-off of variant allele frequency is mandatory to ensure that TML would be a reliable estimation of the number of the mutations in the tumor, avoiding false negative/positive results.

The choice of using a targeted-NGS panel instead of whole-exome sequencing (WES), have been carefully evaluated, since the panel size and the kind of included variants for TML analysis remain a key question [46, 47], and a recent report described that in panels with genomic coverage $<0.5 \mathrm{Mb}$, the accuracy of TML determined by targeted NGS diminished [48]. Nevertheless, our approach was intended to meet the clinic-laboratory need 
of cost-efficiency. Several studies show that TML measured by WES is not currently feasible in routine clinical setting due to high costs, long turnaround time and limited availability of samples [49]. Interesting, previous works demonstrated the comparability of panel-based sequencing versus WES in NSCLC patients treated with immunotherapy: Ritzvi H. et al. [50], performing WES and targeted NGS for 49 patients, showed a significant correlation between the two methodologies $(R=0.86 ; p<0.001)$; similarly, another study by Wang Z. et al. [51] demonstrated the same correlations (median $R^{2}=0.92$; interquartile range $=0.91-0.93$ ). Moreover, the researchers also confirmed that TML may be a potential biomarker to identify patients who will benefit from anti-PD-1/PD-L1 therapy.

The present study was also aimed at evaluating the impact of germline SNPs of PD-L1 (c.-14-368 T > G and c. $* 395 \mathrm{G}>\mathrm{C}$ ) on PFS as previously investigated [9]. In the present work, patients treated with nivolumab showed a shorter median PFS in the c.*395GG wild type carriers, compared with those carrying the mutant c.*395GC/CC genotype. The c.*395G $>\mathrm{C}$ polymorphism increases promoter activity and PD-L1 mRNA levels and is significantly associated with better survival. These findings suggest that PD-L1 polymorphism may be useful predictor of response to treatment [19, 52]. Moreover, our results showed that PD-L1 expression in exosomes is not a predictive biomarker of response at baseline; however, PD-L1 expression was found to increase in patients who progressed to treatment, confirming the results of a previous published study [4]. Given the complexity and multifactorial nature of the anticancer immune response and the mechanisms of tumor immune evasion [38], finding a reliable signature that allows distinguishing patients who will benefit from immunotherapy is still a mission. In this context, radiomics could be a useful tool, as it allows to highlight alterations of neoplastic texture $[53,54]$. Radiomics has the potential to provide an individualized quantitative (and therefore objective) measurement of tissue reaction to treatment in terms of tumor response [17], which cannot be accurately derived by either human visual assessment or laboratory data, alone. However, the significance of the association between radiomics and the complex biological processes occurring within the tumor also remains challenging. Therefore, combined molecular and radiomic data could help to clarify the meaning of imaging-based features and increase the predictive significance of bench results. In our study Pearson's correlation showed a direct relation between exosomal mRNA expression of PD-L1 and radiomic features such as "contrast", "dissimilarity" and "sum variance", while an inverse correlation was showed between PD-L1 expression and "entropy". Moreover, an inverse correlation was also found for PD-L1 expression in tumor tissue with the "autocorrelation" and "sum average" features. Data from this preliminary study show that liquid biopsy can be completed by radiomic features, which could reflect tumor spatial heterogeneity, microenvironment and mutational profile [55]. Although the results emerge from the analysis of 11 patients only, and need to be confirmed in a larger population, they are promising and could be intended to underline the valuable contribution of texture analysis in a multi-parametric context. Our results are consistent with another study from the University of Oklahoma reporting that while radiomics $(\mathrm{AUC}=0.78)$ and genomics $(\mathrm{AUC}=0.78)$ models were capable of predicting survival, accuracy significantly improved $(\mathrm{AUC}=0.84)$ when both data were combined [56].

In the present study a manual segmentation of the VOIs has been adopted, even if it is labour intensive and not always feasible for radiomic analysis, since it requires very large data-sets. Moreover, many authors consider manual segmentation by expert readers the ground truth despite high inter-reader variability [57-60]. However, it is not clear to what degree segmentation variability has an impact on radiomics features, even considering that a universal automatic segmentation algorithm has not been validated and established for all image applications, and some features may not show stability and reproducibility using different methods. Furthermore, automatic segmentation means "probabilistic" segmentation, and the ground truth for automatic boundering comes only for big datasets able to train the neural network [61]. Unfortunately, 11 patients were not sufficient to apply an automatic or semi-automatic approach, leading to more variable results than manual segmentation. The number of enrolled patients for radiomic analysis and the type of lesions to be contoured, however, allowed the use of a handcrafted system for identification and segmentation of primary lesions. Indeed, lung tumours present as homogenous, high-intensity lesions on a background of low-intensity lung parenchyma $[62,63]$ and, therefore, can be manually segmented with high reproducibility and accuracy.

The analysis of different potential biomarkers using sophisticated technologies, represent the major advantages of this study. However, limitations should be also acknowledged: first, some of the examined potential biomarkers have been explored in a limited number of patients, due to the suboptimal yield of genetic material; second, NSCLC patients were included from both the first- and secondline setting, and immune checkpoint inhibitor received was consequently different. Therefore, using anti-PD-1 blockade in different lines of treatment could have been a bias. Nonetheless, multiple studies have investigated the survival benefit of immunotherapy when administered in different lines for patients with NSCLC. As an example, Paz-Ares et al. [64] and Gandhi et al. [65] in 2018, as well as Borghaei et al. in 2019 [66], showed the efficacy of first-line ICIs in both PFS (HR: $0.5695 \%$ CI $0.45-0.70$; HR: 0.52 95\% CI 0.43-0.64; HR: 0.53 95\% CI 0.33-0.86, 
respectively) and OS (HR: 0.64 95\% CI 0.49-0.85; HR: 0.49 95\% CI $0.38-0,64$; HR: $0.5695 \%$ CI $0.32-0.95$, respectively), and similar results also emerged from OS and PFS analysis by Brahmer et al. [67] and Herbst et al. [68] investigating ICIs in the sub-sequent line setting (HR PFS: 0.62 95\% CI $0.47-0.81$ and HR OS: $0.5995 \%$ CI 0.44-0.79; HR PFS: 0.79 95\% CI 0.66-0.94 and HR OS: $0.7195 \%$ CI $0.58-0.88$, respectively). These results suggested that anti-PD-1 inhibitors provide longer PFS and OS both when used as first-line treatment and as a subsequent-line setting, with no substantial differences. Unfortunately, the sample size did not allow to come up with a signature that can be associated with a reliable ORR, and a prospective study with larger population is needed to confirm these preliminary data.

In conclusion, the study of correlations between radiomic features and tumor molecular data may offer a reliable picture of the pathophysiological processes underlying cancer progression, better than single parameters considered individually. The present results confirm the predictive role of a combined approach using genomic and imaging-based data to capture both disease heterogeneity and dynamic changes induced by treatment, suggesting a novel approach in patient management.

If validated in larger and prospective studies, the immune-radiogenomic analysis may thus help in understanding of the molecular determinants of response to immunotherapy.

\section{Acknowledgements None.}

Author contributions MDR and RD conceived and planned the study. IP, GP, NG, CP, LB, AF enrolled the subjects and drawn blood samples. EN, MG, FC acquired radiological imaging. MDR, ER, LF, RC performed the NGS analysis. MDR, ER, SC developed and verified the analytical methods. EN, MG, FC developed computational approaches. All authors discussed the results and contributed to the final manuscript.

Funding Open access funding provided by Università di Pisa within the CRUI-CARE Agreement. This research was funded to RD under grant n. 2017NR7W5K (PRIN 2017) from MIUR, Italy.

Data availability The dataset used in the current study is available as unpublished material, if requested. MDR, FC and RD had full access to all the data in the study and takes responsibility for the integrity of the data and the accuracy of the data analysis.

\section{Compliance with ethical standards}

Conflict of interest The authors report no conflict of interest.

Ethics approval and consent to participate The study was approved by the local Ethics Committee and conducted in accordance with the principles of the Declaration of Helsinki. All patients gave their signed informed consent before blood collection and data analysis.
Consent for publication All patients gave their consent for publication.

Open Access This article is licensed under a Creative Commons Attribution 4.0 International License, which permits use, sharing, adaptation, distribution and reproduction in any medium or format, as long as you give appropriate credit to the original author(s) and the source, provide a link to the Creative Commons licence, and indicate if changes were made. The images or other third party material in this article are included in the article's Creative Commons licence, unless indicated otherwise in a credit line to the material. If material is not included in the article's Creative Commons licence and your intended use is not permitted by statutory regulation or exceeds the permitted use, you will need to obtain permission directly from the copyright holder. To view a copy of this licence, visit http://creativecommons.org/licenses/by/4.0/.

\section{References}

1. Nakamura S, Hayashi K, Imaoka Y et al (2017) Intratumoral heterogeneity of programmed cell death ligand-1 expression is common in lung cancer. PLoS ONE 12:e0186192. https://doi. org/10.1371/journal.pone.0186192

2. Mok TSK, Wu Y-L, Kudaba I et al (2019) Pembrolizumab versus chemotherapy for previously untreated, PD-L1-expressing, locally advanced or metastatic non-small-cell lung cancer (KEYNOTE-042): a randomised, open-label, controlled, phase 3 trial. Lancet (Lond Engl) 393:1819-1830. https://doi. org/10.1016/S0140-6736(18)32409-7

3. Cheng C-C, Lin H-C, Tsai K-J et al (2018) Epidermal growth factor induces STAT1 expression to exacerbate the IFNr-mediated PD-L1 axis in epidermal growth factor receptor-positive cancers. Mol Carcinog 57:1588-1598. https://doi.org/10.1002/ mc. 22881

4. Del Re M, Marconcini R, Pasquini G et al (2018) PD-L1 mRNA expression in plasma-derived exosomes is associated with response to anti-PD- 1 antibodies in melanoma and NSCLC. Br J Cancer 118:820-824. https://doi.org/10.1038/bjc.2018.9

5. Jiang X, Wang J, Deng X et al (2019) Role of the tumor microenvironment in PD-L1/PD-1-mediated tumor immune escape. Mol Cancer 18:10. https://doi.org/10.1186/s12943-018-0928-4

6. Omori S, Kenmotsu H, Abe M et al (2018) Changes in programmed death ligand 1 expression in non-small cell lung cancer patients who received anticancer treatments. Int J Clin Oncol 23:1052-1059. https://doi.org/10.1007/s10147-018-1305-4

7. Dudley JC, Lin M-T, Le DT, Eshleman JR (2016) Microsatellite instability as a biomarker for PD-1 blockade. Clin Cancer Res 22:813-820. https://doi.org/10.1158/1078-0432.CCR-15-1678

8. Heeke S, Hofman P (2018) Tumor mutational burden assessment as a predictive biomarker for immunotherapy in lung cancer patients: getting ready for prime-time or not? Transl lung cancer Res 7:631-638. https://doi.org/10.21037/tlcr.2018.08.04

9. Nomizo T, Ozasa H, Tsuji T et al (2017) Clinical impact of single nucleotide polymorphism in PD-L1 on response to nivolumab for advanced non-small-cell lung cancer patients. Sci Rep 7:45124. https://doi.org/10.1038/srep45124

10. Mimura K, Teh JL, Okayama H et al (2018) PD-L1 expression is mainly regulated by interferon gamma associated with JAKSTAT pathway in gastric cancer. Cancer Sci 109:43-53. https ://doi.org/10.1111/cas.13424

11. Mandai M, Hamanishi J, Abiko K et al (2016) Dual faces of ifngamma in cancer progression: a role of PD-L1 induction in the determination of pro- and antitumor immunity. Clin Cancer Res 22:2329-2334. https://doi.org/10.1158/1078-0432.CCR-16-0224 
12. Kowal J, Tkach M, Thery C (2014) Biogenesis and secretion of exosomes. Curr Opin Cell Biol 29:116-125. https://doi. org/10.1016/j.ceb.2014.05.004

13. Kalluri R (2016) The biology and function of exosomes in cancer. J Clin Invest 126:1208-1215. https://doi.org/10.1172/JCI81135

14. Kharaziha P, Ceder S, Li Q, Panaretakis T (2012) Tumor cellderived exosomes: a message in a bottle. Biochim Biophys Acta 1826:103-111. https://doi.org/10.1016/j.bbcan.2012.03.006

15. Ventimiglia LN, Alonso MA (2016) Biogenesis and function of T cell-derived exosomes. Front cell Dev Biol 4:84. https://doi. org/10.3389/fcell.2016.00084

16. Neri E, Del Re M, Paiar F et al (2018) Radiomics and liquid biopsy in oncology: the holons of systems medicine. Insights Imaging 9:915-924. https://doi.org/10.1007/s13244-018-0657-7

17. Trebeschi S, Drago SG, Birkbak NJ et al (2019) Predicting response to cancer immunotherapy using noninvasive radiomic biomarkers. Ann Oncol Off J Eur Soc Med Oncol 30:998-1004. https://doi.org/10.1093/annonc/mdz108

18. Del Re M, Biasco E, Crucitta S et al (2017) The detection of androgen receptor splice variant 7 in plasma-derived exosomal RNA strongly predicts resistance to hormonal therapy in metastatic prostate cancer patients. Eur Urol 71:680-687. https://doi. org/10.1016/j.eururo.2016.08.012

19. Yeo M-K, Choi S-Y, Seong I-O et al (2017) Association of PD-L1 expression and PD-L1 gene polymorphism with poor prognosis in lung adenocarcinoma and squamous cell carcinoma. Hum Pathol 68:103-111. https://doi.org/10.1016/j.humpa th.2017.08.016

20. Del Re M, Tiseo M, Bordi P et al (2017) Contribution of KRAS mutations and c.2369C $>$ T (p.T790M) EGFR to acquired resistance to EGFR-TKIs in EGFR mutant NSCLC: a study on circulating tumor DNA. Oncotarget 8:13611-13619. https://doi. org/10.18632/oncotarget.6957

21. Schulz WL, Tormey CA, Torres R (2015) Computational approach to annotating variants of unknown significance in clinical next generation sequencing. Lab Med 46:285-289. https://doi. org/10.1309/LMWZH57BRWOPR5RQ

22. Milgram S (1967) The small world problem. Psychol Today 2:60-67

23. Menezes MBC, Kim S, Huang R (2017) Constructing a WattsStrogatz network from a small-world network with symmetric degree distribution. PLoS ONE 12:e0179120. https://doi. org/10.1371/journal.pone.0179120

24. Berenguer R, Pastor-Juan MDR, Canales-Vazquez J et al (2018) Radiomics of CT features may be nonreproducible and redundant: influence of CT acquisition parameters. Radiology 288:407-415. https://doi.org/10.1148/radiol.2018172361

25. Alberich-Bayarri A, Hernandez-Navarro R, Ruiz-Martinez E et al (2017) Development of imaging biomarkers and generation of big data. Radiol Med 122:444-448. https://doi.org/10.1007/s1154 7-017-0742-x

26. de Jong EEC, Sanders KJC, Deist TM et al (2019) Can radiomics help to predict skeletal muscle response to chemotherapy in stage IV non-small cell lung cancer? Eur J Cancer 120:107-113. https ://doi.org/10.1016/j.ejca.2019.07.023

27. Villaruz LC, Socinski MA (2013) The clinical viewpoint: definitions, limitations of RECIST, practical considerations of measurement. Clin Cancer Res Off J Am Assoc Cancer Res 19:26292636. https://doi.org/10.1158/1078-0432.CCR-12-2935

28. Clarke JM, Wang X, Ready NE (2015) Surrogate clinical endpoints to predict overall survival in non-small cell lung cancer trials-are we in a new era? Transl lung cancer Res 4:804-808. https://doi.org/10.3978/j.issn.2218-6751.2015.05.03

29. Aguilar EJ, Ricciuti B, Gainor JF et al (2019) Outcomes to firstline pembrolizumab in patients with non-small-cell lung cancer and very high PD-L1 expression. Ann Oncol Off J Eur Soc Med Oncol 30:1653-1659. https://doi.org/10.1093/annonc/mdz288

30. Schoenborn JR, Wilson CB (2007) Regulation of interferon- $\gamma$ during innate and adaptive immune responses. Adv Immunol 96:41-101. https://doi.org/10.1016/S0065-2776(07)96002-2

31. Ni L, Lu J (2018) Interferon gamma in cancer immunotherapy. Cancer Med 7:4509-4516. https://doi.org/10.1002/cam4.1700

32. Zaidi MR (2019) The interferon-gamma paradox in cancer. J Interf Cytokine Res 39:30-38. https://doi.org/10.1089/jir.2018.0087

33. Castro F, Cardoso AP, Gonçalves RM et al (2018) Interferongamma at the crossroads of tumor immune surveillance or evasion. Front Immunol 9:847. https://doi.org/10.3389/fimmu .2018 .00847

34. Abiko K, Matsumura N, Hamanishi J et al (2015) IFN- $\gamma$ from lymphocytes induces PD-L1 expression and promotes progression of ovarian cancer. Br J Cancer 112:1501-1509. https://doi. org/10.1038/bjc.2015.101

35. Shankaran V, Ikeda H, Bruce AT et al (2001) IFNgamma and lymphocytes prevent primary tumour development and shape tumour immunogenicity. Nature 410:1107-1111. https://doi. org/10.1038/35074122

36. Benci JL, Xu B, Qiu Y et al (2016) Tumor interferon signaling regulates a multigenic resistance program to immune checkpoint blockade. Cell 167:1540-1554.e12. https://doi.org/10.1016/j. cell.2016.11.022

37. Camidge DR, Doebele RC, Kerr KM (2019) Comparing and contrasting predictive biomarkers for immunotherapy and targeted therapy of NSCLC. Nat Rev Clin Oncol 16:341-355. https://doi. org/10.1038/s41571-019-0173-9

38. Anichini A, Tassi E, Grazia G, Mortarini R (2018) The non-small cell lung cancer immune landscape: emerging complexity, prognostic relevance and prospective significance in the context of immunotherapy. Cancer Immunol Immunother 67:1011-1022. https://doi.org/10.1007/s00262-018-2147-7

39. Hellmann MD, Nathanson T, Rizvi H et al (2018) Genomic features of response to combination immunotherapy in patients with advanced non-small-cell lung cancer. Cancer Cell 33:843-852.e4. https://doi.org/10.1016/j.ccell.2018.03.018

40. Rizvi NA, Hellmann MD, Snyder A et al (2015) Cancer immunology. Mutational landscape determines sensitivity to PD-1 blockade in non-small cell lung cancer. Science 348:124-128. https:// doi.org/10.1126/science.aaa1348

41. Chen Y-P, Zhang Y, Lv J-W et al (2017) Genomic analysis of tumor microenvironment immune types across 14 solid cancer types: immunotherapeutic implications. Theranostics 7:35853594. https://doi.org/10.7150/thno.21471

42. Jiang T, Chen X, Su C et al (2020) Pan-cancer analysis of ARID1A alterations as biomarkers for immunotherapy outcomes. J Cancer 11:776-780. https://doi.org/10.7150/jca.41296

43. Shen J, Ju Z, Zhao W et al (2018) ARID1A deficiency promotes mutability and potentiates therapeutic antitumor immunity unleashed by immune checkpoint blockade. Nat Med 24:556-562. https://doi.org/10.1038/s41591-018-0012-z

44. Mathur R, Alver BH, San Roman AK et al (2017) ARID1A loss impairs enhancer-mediated gene regulation and drives colon cancer in mice. Nat Genet 49:296-302. https://doi.org/10.1038/ ng. 3744

45. Melendez B, Van Campenhout C, Rorive S et al (2018) Methods of measurement for tumor mutational burden in tumor tissue. Transl lung cancer Res 7:661-667. https://doi.org/10.21037 /tlcr.2018.08.02

46. Fancello L, Gandini S, Pelicci PG, Mazzarella L (2019) Tumor mutational burden quantification from targeted gene panels: major advancements and challenges. J Immunother Cancer 7:183. https ://doi.org/10.1186/s40425-019-0647-4 
47. Stenzinger A, Endris V, Budczies J et al (2020) Harmonization and Standardization of panel-based tumor mutational burden measurement: real-world results and recommendations of the quality in pathology study. J Thorac Oncol 15:1177-1189. https://doi. org/10.1016/j.jtho.2020.01.023

48. Chalmers ZR, Connelly CF, Fabrizio D et al (2017) Analysis of 100,000 human cancer genomes reveals the landscape of tumor mutational burden. Genome Med 9:34. https://doi.org/10.1186/ s13073-017-0424-2

49. Budczies J, Allgäuer M, Litchfield K et al (2019) Optimizing panel-based tumor mutational burden (TMB) measurement. Ann Oncol 30:1496-1506. https://doi.org/10.1093/annonc/mdz205

50. Rizvi H, Sanchez-Vega F, La K et al (2018) Molecular determinants of response to anti-programmed cell death (PD)-1 and antiprogrammed death-ligand 1 (PD-L1) blockade in patients with non-small-cell lung cancer profiled with targeted next-generation sequencing. J Clin Oncol Off J Am Soc Clin Oncol 36:633-641. https://doi.org/10.1200/JCO.2017.75.3384

51. Wang Z, Duan J, Cai S et al (2019) Assessment of blood tumor mutational burden as a potential biomarker for immunotherapy in patients with non-small cell lung cancer with use of a next-generation sequencing cancer gene panel. JAMA Oncol 5:696-702. https://doi.org/10.1001/jamaoncol.2018.7098

52. Yup S, Kju D, Eun J et al (2016) Functional polymorphisms in PD-L1 gene are associated with the prognosis of patients with early stage non-small cell lung cancer. Gene. https://doi. org/10.1016/j.gene.2016.11.007

53. Khorrami M, Prasanna P, Gupta A et al (2019) Changes in CT radiomic features associated with lymphocyte distribution predict overall survival and response to immunotherapy in non-small cell lung cancer. Cancer Immunol Res. https://doi.org/10.1158/23266066.CIR-19-0476

54. Sun R, Limkin EJ, Vakalopoulou M et al (2018) A radiomics approach to assess tumour-infiltrating CD8 cells and response to anti-PD-1 or anti-PD-L1 immunotherapy: an imaging biomarker, retrospective multicohort study. Lancet Oncol 19:1180-1191. https://doi.org/10.1016/S1470-2045(18)30413-3

55. Sala E, Mema E, Himoto $Y$ et al (2017) Unravelling tumour heterogeneity using next-generation imaging: radiomics, radiogenomics, and habitat imaging. Clin Radiol 72:3-10. https://doi. org/10.1016/j.crad.2016.09.013

56. Emaminejad N, Qian W, Guan Y et al (2016) Fusion of quantitative image and genomic biomarkers to improve prognosis assessment of early stage lung cancer patients. IEEE Trans Biomed Eng 63:1034-1043. https://doi.org/10.1109/TBME.2015.2477688

57. Kumar V, Gu Y, Basu S et al (2012) Radiomics: the process and the challenges. Magn Reson Imaging 30:1234-1248. https://doi. org/10.1016/j.mri.2012.06.010
58. Balagurunathan Y, Gu Y, Wang H et al (2014) Reproducibility and prognosis of quantitative features extracted from CT images. Transl Oncol 7:72-87. https://doi.org/10.1593/tlo.13844

59. Parmar C, Rios Velazquez E, Leijenaar R et al (2014) Robust radiomics feature quantification using semiautomatic volumetric segmentation. PLoS ONE 9:e102107

60. Rizzo S, Botta F, Raimondi S et al (2018) Radiomics: the facts and the challenges of image analysis. Eur Radiol Exp 2:36. https ://doi.org/10.1186/s41747-018-0068-z

61. Haarburger C, Müller-Franzes G, Weninger L et al (2020) Radiomics feature reproducibility under inter-rater variability in segmentations of CT images. Sci Rep 10:12688. https://doi. org/10.1038/s41598-020-69534-6

62. Kalef-Ezra J, Karantanas A, Tsekeris P (1999) CT measurement of lung density. Acta radiol 40:333-337. https://doi. org/10.3109/02841859909175564

63. Sofka M, Wetzl J, Birkbeck N et al (2011) Multi-stage learning for robust lung segmentation in challenging CT volumes. Med image Comput Comput Interv MICCAI. Int Conf Med Image Comput Comput Interv 14:667-674. https://doi.org/10.1007/978-3-64223626-6_82

64. Paz-Ares L, Luft A, Vicente D et al (2018) Pembrolizumab plus chemotherapy for squamous non-small-cell lung cancer. N Engl J Med 379:2040-2051. https://doi.org/10.1056/NEJMoa1810865

65. Gandhi L, Rodríguez-Abreu D, Gadgeel S et al (2018) Pembrolizumab plus chemotherapy in metastatic non-small-cell lung cancer. N Engl J Med 378:2078-2092. https://doi.org/10.1056/ NEJMoa1801005

66. Borghaei H, Langer CJ, Gadgeel S et al (2019) 24-Month overall survival from KEYNOTE-021 cohort G: pemetrexed and carboplatin with or without pembrolizumab as first-line therapy for advanced nonsquamous non-small cell lung cancer. J Thorac Oncol Off Publ Int Assoc Study Lung Cancer 14:124-129. https ://doi.org/10.1016/j.jtho.2018.08.004

67. Brahmer J, Reckamp KL, Baas P et al (2015) Nivolumab versus docetaxel in advanced squamous-cell non-small-cell lung cancer. N Engl J Med 373:123-135. https://doi.org/10.1056/NEJMoa1504 627

68. Herbst RS, Baas P, Kim D-W et al (2016) Pembrolizumab versus docetaxel for previously treated, PD-L1-positive, advanced nonsmall-cell lung cancer (KEYNOTE-010): a randomised controlled trial. Lancet (Lond Engl) 387:1540-1550. https://doi.org/10.1016/ S0140-6736(15)01281-7

Publisher's Note Springer Nature remains neutral with regard to jurisdictional claims in published maps and institutional affiliations.

\section{Affiliations}

\section{Marzia Del Re ${ }^{1}$. Federico Cucchiara ${ }^{1}$ - Eleonora Rofi ${ }^{1}$. Lorenzo Fontanelli ${ }^{1}$ - lacopo Petrini ${ }^{2} \cdot$ Nicole Gri $^{3}$. Giulia Pasquini $^{2} \cdot$ Mimma Rizzo $^{3} \cdot$ Michela Gabelloni $^{4} \cdot$ Lorenzo Belluomini $^{5} \cdot$ Stefania Crucitta ${ }^{1} \cdot$ Raffaele Ciampi $^{6}$. Antonio Frassoldati ${ }^{5} \cdot$ Emanuele Neri $^{4} \cdot$ Camillo Porta $^{3,7,8} \cdot$ Romano Danesi $^{1}$ (i)}

1 Unit of Clinical Pharmacology and Pharmacogenetics, Department of Clinical and Experimental Medicine, University of Pisa, Pisa, Italy

2 General Pathology, Department of Translational Research and New Technologies in Medicine, University of Pisa, Pisa, Italy
3 Division of Translational Oncology, IRCCS Istituti Clinici Scientifici Maugeri, Pavia, Italy

4 Diagnostic and Interventional Radiology, Department of Translational Research and New Technologies in Medicine, University of Pisa, Pisa, Italy

5 Unit of Clinical Oncology, Specialist Medical Department, S. Anna University Hospital, Ferrara, Italy 
6 Endocrinology Unit, Department of Clinical and Experimental Medicine, University of Pisa, Pisa, Italy

7 Department of Internal Medicine and Therapeutics, University of Pavia, Pavia, Italy
8 Present Address: Unit of Medical Oncology, Department of Biomedical Sciences and Human Oncology, University of Bari 'A. Moro', Bari, Italy 